\title{
Tumor Targeting Using Affibody Molecules: Interplay of Affinity, Target Expression Level, and Binding Site Composition
}

\author{
Vladimir Tolmachev ${ }^{1}$, Thuy A. Tran ${ }^{1,2}$, Daniel Rosik ${ }^{3}$, Anna Sjöberg ${ }^{4}$, Lars Abrahmsén $^{5}$, and Anna Orlova ${ }^{6}$ \\ ${ }^{I}$ Department of Biomedical Radiation Sciences, Uppsala University, Uppsala, Sweden; ${ }^{2}$ Lund University Bioimaging Center, \\ Lund University, Lund, Sweden; ${ }^{3}$ School of Biotechnology, Royal Institute of Technology, Stockholm, Sweden; ${ }^{4}$ Atlas Antibodies AB, \\ AlbaNova University Center, Stockholm, Sweden; ${ }^{5}$ Algeta ASA, Oslo, Norway; and ${ }^{6}$ Preclinical PET Platform, Department of \\ Medicinal Chemistry, Uppsala University, Uppsala, Sweden
}

Radionuclide imaging of cancer-associated molecular alterations may contribute to patient stratification for targeting therapy. Scaffold high-affinity proteins, such as Affibody molecules, are a new, promising class of probes for in vivo imaging. Methods. The effects of human epidermal growth factor receptor 2 (HER2) affinity and binding site composition of HER2-binding Affibody molecules, and of the HER2 density on the tumor targeting, were studied in vivo. The tumor uptake and tumor-to-organ ratios of Affibody molecules with moderate (dissociation constant $\left[\mathrm{K}_{\mathrm{D}}\right]=$ $\left.10^{-9} \mathrm{M}\right)$ or high $\left(\mathrm{K}_{\mathrm{D}}=10^{-10} \mathrm{M}\right)$ affinity were compared between tumor xenografts with a high (SKOV-3) and low (LS174T) HER2 expression level in BALB/C nu/nu mice. Two Affibody molecules with similar affinity $\left(K_{D}=10^{-10} \mathrm{M}\right)$ but having alternative amino acids in the binding site were compared. Results. In SKOV-3 xenografts, uptake was independent of affinity at $4 \mathrm{~h}$ after injection, but high-affinity binders provided 2-fold-higher tumor radioactivity retention at $24 \mathrm{~h}$. In LS174T xenografts, uptake of high-affinity probes was already severalfold higher at $4 \mathrm{~h}$ after injection, and the difference was increased at $24 \mathrm{~h}$. The clearance rate and tumor-to-organ ratios were influenced by the amino acid composition of the binding surface of the tracer protein. Conclusion. The optimal affinity of HER2-binding Affibody molecules depends on the expression of a molecular target. At a high expression level ( $>10^{6}$ receptors per cell), an affinity in the low-nanomolar range is sufficient. At moderate expression, subnanomolar affinity is desirable. The binding site composition can influence the imaging contrast. This information may be useful for development of imaging agents based on scaffold affinity proteins.

Key Words: radionuclide molecular imaging; scaffold proteins; Affibody molecules; affinity; HER2

J Nucl Med 2012; 53:953-960

DOI: 10.2967/jnumed.111.101527

\footnotetext{
Received Dec. 3, 2011; revision accepted Feb. 28, 2012.

For correspondence or reprints contact: Vladimir Tolmachev, Department of Biomedical Radiation Sciences, Uppsala University, SE-751 85 Uppsala, Sweden.

E-mail: vladimir.tolmachev@bms.uu.se

Published online May 14, 2012.

COPYRIGHT @ 2012 by the Society of Nuclear Medicine, Inc.
}

$\mathbf{R}$ adionuclide imaging of molecular drug targets is a potential means of stratifying patients for targeted cancer therapy. A precondition for successful implementation of radionuclide molecular imaging into clinical practice is high sensitivity. The sensitivity is determined by contrast, which depends on the ratio of radioactivity concentrations in the tumor and in surrounding healthy tissues (tumor-toorgan ratios). Thus, when developing new imaging agents, the focus is on factors increasing these ratios, both by increasing tumor uptake and retention of radioactivity and by decreasing uptake in healthy tissues. Some cancer-related molecular targets, such as receptor tyrosine kinases (1) and $\mathrm{G}$ protein-coupled receptors (2), are expressed on the cell surface, enabling the use of protein-binding targeting agents as imaging probes for patient stratification. The use of small targeting proteins and peptides instead of antibodies enables increased sensitivity due to reduction of background, and enhanced specificity by avoiding non-target-specific tumor accumulation resulting from the enhanced permeability and retention effect (3).

In vitro molecular display of collections of binding proteins facilitates selection of high-affinity binders, including proteins smaller than the smallest antibody fragments. Several types of proteins have been used as scaffolds to create collections ("libraries") of potential binders (4). A suitable scaffold protein contains a robust structurally defined framework and a spatially defined surface area that may be more or less randomly modified. Several classes of scaffold protein-based radiolabeled probes have a high potential for in vivo molecular imaging, including Affibody molecules (Affibody AB), cystine knot peptides, and nanobodies (5). Affibody molecules are small $(6 \mathrm{kDa})$, robust 3-helix proteins, based on a modified B-domain of staphylococcal protein A. Randomization of 13 amino acids on a surface made up of residues from helices 1 and 2 has been done to construct large $\left(>10^{10}\right.$ members) libraries (6). Affibody molecules having high-affinity binding to selected therapeutic targets have been developed-for example, human epidermal growth factor receptor 2 (HER2), epidermal growth factor receptor, insulinlike growth factor 1 , 
and platelet-derived growth factor receptor $\beta(7-10)$. Preclinical studies have demonstrated favorable properties of radiolabeled Affibody molecules for in vivo imaging, including high contrast only a few hours after injection (11). HER2binding Affibody molecules labeled with ${ }^{111} \mathrm{In}$ or ${ }^{68} \mathrm{Ga}$ have also been shown to image HER2-expressing metastases in breast cancer patients (12).

Affinity, a measure of the strength of interaction between the targeting agent and its target, is a crucial parameter defining the efficacy of tumor targeting alongside extravasation and clearance rates, diffusion rate in a tumor interstitium, and the differential presence of the target in tumor and healthy tissues $(13,14)$. Internalization of the targeting protein followed by metabolism and intracellular trapping of residualizing labels, such as radiometals, might make cellular binding of targeting proteins and peptides less dependent on their affinity (15). However, internalization of HER2-binding Affibody molecules is relatively slow, typically around $30 \%$ of bound conjugate per day, and in this case cellular retention of radioactivity results from the strong target binding (16-18). A high affinity to HER2 is therefore essential for high tumor accumulation of Affibody-associated radioactivity.

Several studies on the influence of affinity of single-chain variable fragments, diabodies, and full-length IgG on tumor targeting have been performed (19-21). The results of these studies suggest that an affinity increase beyond $10^{-9} \mathrm{M}$ does not offer increased tumor uptake. A "binding site barrier" (i.e., retardation of antibody penetration in tumor due to interaction with antigen in the outer layers of the cells in tumors) has been suggested as a limiting factor for tumor uptake in the case of very high affinity (22). The targeting proteins used in these studies are much larger than Affibody molecules and have different rates of blood clearance, extravasation, and diffusion. For this reason, the results cannot be directly translated to Affibody molecules. Furthermore, models having tumors displaying a high level of the molecular target (over 1 million copies per cell) were used in these studies. However, imaging of tumors with a lower level of target display might also be required. For example, detection of moderate overexpression of HER2 in prostate cancer can provide important prognostic and predictive information (23).

Several studies have shown that small changes at surfaceexposed positions of the Affibody molecule can have a large impact on biodistribution $(24,25)$, especially on hepatic and renal uptake. It is therefore reasonable to postulate that the composition of the binding site may influence the pharmacokinetic properties of an Affibody molecule. In this study, the effects of HER2 affinity and binding site composition of
HER2-binding Affibody molecules, and of the HER2 density, on the tumor were studied in vivo. First, the tumor uptake and tumor-to-organ ratios of Affibody molecules binding HER2 with moderate (dissociation constant $\left[K_{D}\right]=10^{-9} \mathrm{M}$ ) or high $\left(K_{D}=10^{-10} \mathrm{M}\right)$ affinity were investigated in tumor xenografts with high and low HER2 expression level. Second, the biodistribution was compared between 2 Affibody molecules having similar affinity for HER2 but alternative amino acids in the binding site.

\section{MATERIALS AND METHODS}

\section{Design, Synthesis, and Characterization of Affibody Molecules}

Three Affibody molecules with different affinities to HER2 ( 2 with low-picomolar and 1 with low-nanomolar affinity) were selected among variants created during affinity maturation (7). The amino acid sequences of these Affibody molecules are identical, except for a few positions in the binding site (i.e., positions $9,11,17,18,24,25$, and 27) providing a difference in affinity to HER2 (Fig. 1). The Affibody molecules were assembled using the Fmoc strategy on a fully automated peptide synthesizer and purified as described earlier (18). To provide site-specific conjugation, a unique cysteine was introduced at position 59. The maleimido derivative of DOTA chelator [MMA-DOTA] was conjugated to the cysteine thiol, as described earlier (17), to enable site-specific radiolabeling with ${ }^{111}$ In. The DOTA-conjugated Affibody molecules were denoted as PEP05541, PEP05838, and PEP7127. The identity of the conjugates was confirmed and the purity evaluated by high-performance liquid chromatography and online mass spectrometry (HPLC-MS) using an Agilent 1100 Series LC/ MSD equipped with electrospray ionization and a single quadrupole as described earlier (18). HER2 binding analysis was performed on a Biacore 2000 instrument (GE Healthcare) as described by Tran et al. (18).

The negative control was $\mathrm{Z}_{\text {Taq }}$, an Affibody molecule binding Taq-polymerase but not HER2. This protein was conjugated with DOTA and labeled in the same way.

\section{Labeling and Stability}

DOTA-conjugated Affibody molecule $(40 \mu \mathrm{L}, 1 \mathrm{mg} / \mathrm{mL}$ in $0.2 \mathrm{M}$ ammonium acetate, $\mathrm{pH} 5.5$ ) was mixed with $16 \mu \mathrm{L}$ of $0.2 \mathrm{M}$ ammonium acetate, $\mathrm{pH} 5.5$, and $54 \mu \mathrm{L}$ (20 MBq) of ${ }^{111} \mathrm{In}$ (Tyco Healthcare) in $0.05 \mathrm{M} \mathrm{HCl}$. The mixture was incubated at $60^{\circ} \mathrm{C}$ during $30 \mathrm{~min}$ and analyzed using instant thin-layer chromatography (150-771 dark green Tec-Control chromatography strips; Biodex Medical Systems). For quality control of the labeling, instant thin-layer chromatography strips were eluted in $0.2 \mathrm{M}$ citric acid. Distribution of radioactivity was analyzed using a Cyclone Storage Phosphor System (Perkin Elmer). To evaluate the labeling stability, the radiolabeled peptides ( 2 samples for each) were incubated for $4 \mathrm{~h}$ at room temperature with a 500-fold molar excess of ethylenediamine tetraacetic acid and analyzed using instant thin-layer chromatography.
FIGURE 1. Alignment of studied HER2binding Affibody molecules. Amino acids that differ in studied variants are red.
PEP05541: AEAKY AKEMR NAYWE IALLP NLTNQ QKRAF IRKLY DDPSQ SSELL SEAKK LNDSQ GSECG [C ${ }^{59}$-mal DOTA] PEP05838: AEAKY AKEFR TAYWE IAVLP NLTNQ QKRAF IRKLY DDPSQ SSELL SEAKK LNDSQ GSECG [C ${ }^{59}$-mal DOTA] PEP07127: AEAKY AKELR TAYWE IVSLP NLTRN QSRAF IRKLY DDPSQ SSELL SEAKK LNDSQ GSECG [ ${ }^{59}$-mal DOTA] 10
20
40 


\section{In Vitro Binding Specificity and Cellular Processing of Affibody Molecules}

The HER2-expressing ovarian carcinoma SKOV-3 (with a high level of HER2 expression) and colorectal carcinoma LS174T (with a low level of HER2 expression) cell lines (both from American Type Culture Collection) were used for studies of binding specificity and cellular processing. Cells were cultured in RPMI medium (Flow Irvine), supplemented with $10 \%$ fetal calf serum (Sigma), $2 \mathrm{mM}$ L-glutamine, and PEST (penicillin, $100 \mathrm{IU} / \mathrm{mL}$, and streptomycin, $100 \mu \mathrm{g} / \mathrm{mL}$; Biokrom Kg).

Expression of HER2 in these cell lines was quantitatively determined by a saturation assay (Supplemental data, available online only at http://jnm.snmjournals.org) as described earlier (26).

In vitro binding and processing experiments with radiolabeled Affibody molecules were performed according to a method developed and validated earlier (Supplemental data) (16).

\section{Biodistribution Studies}

The animal studies were approved by the Local Ethics Committee for Animal Research. Tumors were grafted in the right hind leg on female outbred BALB/c nu/nu mice by subcutaneous injection of $1 \times 10^{7}$ SKOV-3 or $1 \times 10^{6}$ LS174T cells. Tumors were allowed to develop during 6 or 3 wk for SKOV-3 xenografts and LS174T xenografts, respectively. At the time of the experiment, the average tumor weight was $0.35 \pm 0.21 \mathrm{~g}$ for LS174T and $0.18 \pm 0.01 \mathrm{~g}$ for SKOV-3 xenografts.

For each type of xenograft, 7 groups of mice (6-8 animals each) were used. Two groups of mice were injected with each specific conjugate (40 kBq in $100 \mu \mathrm{L}$ of phosphate-buffered saline, $1 \mu \mathrm{g}$ / $0.13 \mathrm{nmol}$ per animal) and sacrificed at 4 or $24 \mathrm{~h}$ after injection. One group was injected with the same dose of control non-HER2binding ${ }^{111} \mathrm{In}-\mathrm{Z}_{\mathrm{Taq}}$ Affibody molecule, and tumor uptake was measured at $4 \mathrm{~h}$ after injection. Mice was euthanized by overdosing of anesthesia ( $20 \mu \mathrm{L}$ of solution per gram of body weight; ketamine, $10 \mathrm{mg} / \mathrm{mL}$; xylazine, $1 \mathrm{mg} / \mathrm{mL}$ ) before heart puncture with a heparinized syringe and withdrawal of blood. Blood and organ samples, as well as the remainder of the carcass, were collected and their radioactivity was measured. The organ uptake values were calculated as percentage of injected activity per gram of tissue except for the gastrointestinal tract with its content (which was used as a measure of hepatobiliary excretion of radioactivity) and the remainder of the carcass, which were calculated as percentage injected activity per whole sample.

\section{Statistics}

Data on cellular uptake and biodistribution were analyzed by an unpaired, 2-tailed $t$ test using Prism 5 software (GraphPad Software) to determine any significant differences $(P<0.05)$.

\section{RESULTS}

\section{Design, Synthesis, and Characterization of} Affibody Molecules

The purity of the Affibody molecules was more than $97 \%$. The identity of the synthetic Affibody molecules was confirmed by high-performance liquid chromatography and online mass spectrometry (Supplemental Fig. 1). The binding kinetics of the DOTA-conjugated peptides to HER2 were measured by surface plasmon resonance (sensorgrams in Supplemental Fig. 2), and the binding affinity $\left(\mathrm{K}_{\mathrm{D}}\right)$ of the peptides to HER2 protein was calculated from dissociation and association constants (Table 1). The dissociation constants at equilibrium were $116.7 \pm 0.10 \mathrm{pM}, 157 \pm 4 \mathrm{pM}$, and 3,804 \pm 178 pM for PEP05541, PEP05838, and PEP07127, respectively.

\section{Labeling, in Vitro Binding Specificity, and Cellular Processing of Affibody Molecules}

The labeling yields were higher than $99 \%$. For this reason, the radiolabeled Affibody molecules were used for experiments without additional purification. A specific radioactivity of $3.5 \mathrm{GBq} / \mu \mathrm{mol}$ was obtained for all Affibody molecules. Challenge with a 500-fold excess of ethylenediamine tetraacetic acid caused a marginal release of radioactivity $(0.5 \%-$ $1.5 \%$ ) from the conjugates, indicating that the labeling was stable (Supplemental Table 1).

The results of the saturation assay showed that SKOV-3 cells express $(1.63 \pm 0.08) \cdot 10^{6}$ receptors per cell, and LS174T cells express $(3.9 \pm 0.6) \cdot 10^{4}$ receptors per cell (Supplemental Fig. 3).

In the in vitro binding specificity experiment, presaturation of HER2 receptors in SKOV-3 cells with the unlabeled Affibody molecule $\mathrm{Z}_{\mathrm{HER}: 342}$ significantly reduced $(P<$ $10^{-6}$ ) the cell-bound activity of all conjugates (Supplemental Fig. 4). Presaturation of cells with the unlabeled control Affibody molecule $Z_{\text {EGFR:1864 (binding the epidermal }}$ growth factor receptor) (8) did not influence binding of anti-HER2 conjugates. These results, showing that the binding to HER2-expressing cells was saturable, support that all HER2 binders in the study bind the same site.

The cellular processing analysis in SKOV-3 cells showed that the internalization of all indium-labeled conjugates was equally slow. After a 4-h incubation, only $12.5 \% \pm$ $0.9 \%, 11.9 \% \pm 0.5 \%$, and $12.8 \% \pm 0.6 \%$ of cell-bound

TABLE 1

Binding Kinetics and Affinity of the Affibody Molecules Determined by Surface Plasmon Resonance

\begin{tabular}{cccc}
\hline Molecule & $\mathrm{k}_{\mathrm{a}}(1 / \mathrm{Ms})$ & $\mathrm{k}_{\mathrm{d}}(1 / \mathrm{s})$ & $\mathrm{K}_{\mathrm{D}}(\mathrm{pM})$ \\
\hline PEP05541 & $(6.3 \pm 1.1) \times 10^{6}$ & $(7.37 \pm 1.33) \times 10^{-4}$ & $116.7 \pm 0.1$ \\
PEP05838 & $(9.2 \pm 1.8) \times 10^{6}$ & $(1.43 \pm 0.25) \times 10^{-3}$ & $157 \pm 4$ \\
PEP07127 & $(6.69 \pm 0.01) \times 10^{6}$ & $(2.55 \pm 0.12) \times 10^{-2}$ & $3804 \pm 178$
\end{tabular}

$\mathrm{k}_{\mathrm{a}}=$ association rate constant; $\mathrm{k}_{\mathrm{b}}=$ dissociation rate constant; $\mathrm{K}_{\mathrm{D}}=$ dissociation constant at equilibrium.

High affinity corresponds to small $K_{D}$ values. 
radioactivity were internalized for ${ }^{111} \mathrm{In}$-PEP05541, ${ }^{111} \mathrm{In}-$ PEP05838, and ${ }^{111}$ In-PEP07127, respectively. Internalization increased after a $24-\mathrm{h}$ incubation to $36.5 \% \pm 0.6 \%$, $37.6 \% \pm 0.1 \%$, and $35 \% \pm 1 \%$ for ${ }^{111} \mathrm{In}-\mathrm{PEP} 05541,{ }^{111} \mathrm{In}-$ PEP05838, and ${ }^{111}$ In-PEP07127, respectively.

\section{Biodistribution Studies}

Data concerning biodistribution of ${ }^{111}$ In-PEP05541, ${ }^{111}$ In-PEP05838, and ${ }^{111}$ In-PEP07127 in mice bearing tumor xenografts with different levels of HER2 expression are presented in Tables 2 and 3. The general biodistribution pattern was similar for all conjugates. All were rapidly cleared from blood and from most normal organs and tissues. All yielded a low radioactivity accumulation in the gastrointestinal tract but high accumulation in the kidneys. However, significant differences were seen in accumulation of radioactivity in normal organs and tissues for different conjugates. Overall, it was the lowest with ${ }^{111}$ In-PEP07127. Furthermore, higher levels of radioactivity in liver and spleen were obtained with ${ }^{111} \mathrm{In}$ PEP05838 than with the other 2 variants.

For all conjugates, the radioactivity accumulating in the tumor in either tumor model exceeded the level in any organ or tissue, except the kidneys. Figure 2 shows that only HER2-specific Affibody molecules-not the non-HER2binding control-are taken up in the tumor in either type of xenograft. Uptake of specific targeting agents was significantly higher than uptake of the non-tumor-specific control $(P<0.0001)$.

At $4 \mathrm{~h}$ after injection, there was no significant difference between the high-affinity conjugates $\left({ }^{111} \mathrm{In}\right.$-PEP05541 and ${ }^{111}$ In-PEP05838) and the low-affinity conjugate ${ }^{111} \mathrm{In}$ PEP07127 in SKOV-3 xenografts having a high level of HER2 expression (Table 2). However at $24 \mathrm{~h}$ after injection, ${ }^{111}$ In-PEP05541 and ${ }^{111}$ In-PEP05838 gave a more than 2- fold higher level of radioactivity in the SKOV-3 tumor, compared with ${ }^{111}$ In-PEP07127. In contrast, the affinity of the conjugates had a pronounced effect on the level of radioactivity accumulating in the low-HER2-expressing LS174T tumors. Already at $4 \mathrm{~h}$ after injection, the level of radioactivity in the tumor was 4.3- to 6.3-fold higher with the high-affinity constructs than with ${ }^{111}$ In-PEP07127 (Table 3). The difference increased further at $24 \mathrm{~h}$ after injection and was more pronounced at this time point in ${ }^{111}$ In-PEP07127 xenografts than in SKOV-3 xenografts.

The data concerning tumor-to-organ ratios are presented in Figures 3 and 4 . At $4 \mathrm{~h}$ after injection, ${ }^{111} \mathrm{In}-\mathrm{PEP} 07127$ provided higher tumor-to-organ ratios than the high-affinity conjugates in SKOV-3 xenografts. Tumor-to-blood ratio was equal for ${ }^{111} \mathrm{In}$-PEP07127 and ${ }^{111} \mathrm{In}$-PEP05838, but tumor-to-liver, tumor-to-lung, and tumor-to-muscle ratios were superior with the low-affinity ${ }^{111}$ In-PEP07127. Somewhat slower blood clearance of ${ }^{111}$ In-PEP05541 resulted in lower tumor-to-blood ratios than were found for the 2 other constructs. The pattern was changed at $24 \mathrm{~h}$ after injection: With the high-affinity constructs, the tumor-associated (decay-corrected) radioactivity was not changed significantly from the values at $4 \mathrm{~h}$ after injection, whereas only half remained with ${ }^{111} \mathrm{In}$-PEP07127. At $24 \mathrm{~h}$, the tumor-to-organ ratios for high-affinity conjugates were equal or exceeded the corresponding values for ${ }^{111}$ In-PEP07127.

The pattern of tumor-to-organ ratios was very different in the case of LS174T xenografts having low HER2 expression (Fig. 4). At $4 \mathrm{~h}$ after injection, the high-affinity conjugates already provided much higher values than the low-affinity ${ }^{111}$ In-PEP07127. Differences were apparent between the 2 high-affinity constructs in that the tumor-to-blood and tumorto-lung ratios were higher for ${ }^{111}$ In-PEP05838, whereas the tumor-to-liver ratio was higher for ${ }^{111}$ In-PEP05541.

TABLE 2

Biodistribution in BALB/C nu/nu Mice Bearing SKOV-3 Xenografts (High Level of HER2 Expression)

\begin{tabular}{|c|c|c|c|c|c|c|}
\hline \multirow[b]{2}{*}{ Tissue } & \multicolumn{3}{|c|}{$4 \mathrm{~h}$} & \multicolumn{3}{|c|}{$24 \mathrm{~h}$} \\
\hline & ${ }^{111}$ In-PEP05541 & ${ }^{111}$ In-PEP05838 & ${ }^{111}$ In-PEP07127 & ${ }^{111}$ In-PEP05541 & ${ }^{111}$ In-PEP05838 & ${ }^{111}$ In-PEP07127 \\
\hline Blood & $0.23 \pm 0.03^{\dagger \ddagger}$ & $0.13 \pm 0.02$ & $0.11 \pm 0.02$ & $0.09 \pm 0.02^{\dagger \ddagger}$ & $0.07 \pm 0.01$ & $0.06 \pm 0.01$ \\
\hline Lung & $0.33 \pm 0.09 \ddagger$ & $0.36 \pm 0.08^{\S}$ & $0.18 \pm 0.02$ & $0.22 \pm 0.06$ & $0.26 \pm 0.01$ & $0.21 \pm 0.11$ \\
\hline Liver & $1.2 \pm 0.2^{\dagger \ddagger}$ & $2.1 \pm 0.5^{\S}$ & $0.69 \pm 0.10$ & $1.0 \pm 0.2^{\dagger \ddagger}$ & $1.7 \pm 0.3^{\S}$ & $0.6 \pm 0.2$ \\
\hline Spleen & $0.35 \pm 0.06^{\dagger \ddagger}$ & $0.46 \pm 0.09 \S$ & $0.27 \pm 0.03$ & $0.41 \pm 0.13$ & $0.54 \pm 0.09 \S$ & $0.35 \pm 0.15$ \\
\hline Kidney & $224 \pm 24$ & $231 \pm 2$ & $253 \pm 31$ & $178 \pm 31$ & $180 \pm 30$ & $178 \pm 43$ \\
\hline Tumor & $12.9 \pm 2.8$ & $15.5 \pm 6.6$ & $14.3 \pm 1.2$ & $15.9 \pm 3.9^{\ddagger}$ & $15.5 \pm 3.9^{\S}$ & $7.1 \pm 2.4$ \\
\hline Muscle & $0.04 \pm 0.02^{\dagger}$ & $0.1 \pm 0.02^{\S}$ & $0.05 \pm 0.01$ & $0.08 \pm 0.03^{\dagger}$ & $0.12 \pm 0.02^{\S}$ & $0.06 \pm 0.01$ \\
\hline Bone & $0.21 \pm 0.03^{\dagger}$ & $0.28 \pm 0.07 \S$ & $0.14 \pm 0.03$ & $0.3 \pm 0.0^{\dagger}$ & $0.4 \pm 0.1^{\S}$ & $0.2 \pm 0.1$ \\
\hline Gastrointestinal tract* & $1.3 \pm 0.8$ & $1.2 \pm 0.3^{\S}$ & $0.9 \pm 0.2$ & $0.5 \pm 0.1$ & $0.6 \pm 0.1$ & $0.4 \pm 0.1$ \\
\hline Carcass* & $2.9 \pm 0.6^{\dagger \ddagger}$ & $3.9 \pm 0.8^{\S}$ & $2.1 \pm 0.2$ & $2.5 \pm 0.5^{\ddagger}$ & $2.9 \pm 0.5^{\S}$ & $1.5 \pm 0.4$ \\
\hline
\end{tabular}

Data are expressed as percentage injected activity per gram of tissue and presented as average $(n=8)$ and SD.

*Data for gastrointestinal tract (with content) and carcass are presented as percentage injected activity per whole sample.

†Significant difference $(P<0.05)$ between PEP05541 and PEP05838.

${ }^{\ddagger}$ Significant difference $(P<0.05$; $t$ test) between PEP05541 and PEP07127.

§Significant difference $(P<0.05$; $t$ test) between PEP05838 and PEP07127. 
TABLE 3

Biodistribution in BALB/C nu/nu Mice Bearing LA147T Xenografts (Low Level of HER2 Expression)

\begin{tabular}{|c|c|c|c|c|c|c|}
\hline \multirow[b]{2}{*}{ Tissue } & \multicolumn{3}{|c|}{$4 \mathrm{~h}$} & \multicolumn{3}{|c|}{$24 \mathrm{~h}$} \\
\hline & ${ }^{111}$ In-PEP05541 & ${ }^{111}$ In-PEP05838 & ${ }^{111}$ In-PEP07127 & ${ }^{111}$ In-PEP05541 & ${ }^{111}$ In-PEP05838 & ${ }^{111} \mid \mathrm{n}-\mathrm{PEP} 07127$ \\
\hline Blood & $0.29 \pm 0.06^{\dagger}$ & $0.17 \pm 0.03^{\S}$ & $0.14 \pm 0.02$ & $0.10 \pm 0.02^{\dagger \ddagger}$ & $0.06 \pm 0.01^{\S}$ & $0.05 \pm 0.01$ \\
\hline Lung & $0.38 \pm 0.06^{\ddagger}$ & $0.43 \pm 0.04 \S$ & $0.24 \pm 0.03$ & $0.18 \pm 0.03^{\ddagger}$ & $0.23 \pm 0.04$ & $0.10 \pm 0.01$ \\
\hline Liver & $1.3 \pm 0.3^{\dagger \neq}$ & $2.4 \pm 0.2^{\S}$ & $0.8 \pm 0.1$ & $0.94 \pm 0.21^{\dagger}$ & $1.91 \pm 0.29 \S$ & $0.67 \pm 0.08$ \\
\hline Spleen & $0.41 \pm 0.10^{\dagger \ddagger}$ & $0.58 \pm 0.07 \S$ & $0.30 \pm 0.04$ & $0.35 \pm 0.06^{\dagger}$ & $0.56 \pm 0.08^{\S}$ & $0.24 \pm 0.07$ \\
\hline Kidney & $254 \pm 12$ & $237 \pm 29$ & $265 \pm 26$ & $191 \pm 31^{\ddagger}$ & $194 \pm 18^{\S}$ & $224 \pm 19$ \\
\hline Tumor & $7.4 \pm 1.60^{\dagger \ddagger}$ & $10.8 \pm 1.3^{\S}$ & $1.71 \pm 0.24$ & $3.29 \pm 0.28^{\dagger \ddagger}$ & $4.94 \pm 0.82^{\S}$ & $0.44 \pm 0.07$ \\
\hline Muscle & $0.12 \pm 0.05^{\ddagger}$ & $0.13 \pm 0.02^{\S}$ & $0.09 \pm 0.02$ & $0.07 \pm 0.04$ & $0.09 \pm 0.02$ & $0.05 \pm 0.02$ \\
\hline Bone & $0.31 \pm 0.08^{\dagger \ddagger}$ & $0.41 \pm 0.08 \S$ & $0.19 \pm 0.03$ & $0.21 \pm 0.04$ & $0.28 \pm 0.06$ & $0.15 \pm 0.02$ \\
\hline Gastrointestinal tract ${ }^{\star}$ & $1.1 \pm 0.3$ & $1.1 \pm 0.4$ & $1.1 \pm 0.3$ & $0.53 \pm 0.14$ & $0.56 \pm 0.06$ & $0.44 \pm 0.08$ \\
\hline Carcass $^{*}$ & $3.5 \pm 0.7^{\dagger \ddagger}$ & $5.0 \pm 1.4 \S$ & $3.0 \pm 0.4$ & $2.37 \pm 0.32^{\dagger \ddagger}$ & $2.90 \pm 0.39$ & $1.71 \pm 0.09$ \\
\hline \multicolumn{7}{|c|}{$\begin{array}{l}\text { Data are expressed as percentage injected activity per gram of tissue and presented as average }(n=6) \text { and SD. } \\
{ }^{*} \text { Data for gastrointestinal tract (with content) and carcass are presented as percentage injected activity per whole sample. } \\
{ }^{\dagger} \text { Significant difference }(P<0.05) \text { between PEP05541 and PEP05838. } \\
\text { †Significant difference }(P<0.05) \text { between PEP05541 and PEP07127. } \\
\text { \$S ignificant difference }(P<0.05) \text { between PEP05838 and PEP0712. }\end{array}$} \\
\hline
\end{tabular}

\section{DISCUSSION}

In this study, the influence of small differences in the binding site on biodistribution and tumor targeting was investigated using a panel of 3 HER2-binding Affibody molecules, including 2 binders with high affinity $\left(\mathrm{K}_{\mathrm{D}}, \sim 10^{-10} \mathrm{M}\right)$ and 1 with moderate affinity $\left(\mathrm{K}_{\mathrm{D}}, 10^{-9} \mathrm{M}\right)$. All size-related factors, such as extravasation rate, rate of diffusion in extracellular space, and glomerular filtration rate, are expected to be identical for these 3 tracers. All 3 protein molecules were site-specifically labeled with ${ }^{111} \mathrm{In}$ at a C-terminally located macrocyclic chelator DOTA, providing an extremely stable attachment of the radiometal nuclide to the protein due to exceptional kinetic inertness of the chelate (27). Therefore, no factors related to labeling, such as a potential release of nuclide in circulation (if any) or modification of the overall lipophilicity, should have influenced the results. Furthermore, in vitro internalization experiments confirmed that the rate of internalization was equally slow with all 3 variants; yielding $12 \%-13 \%$ of cell-bound activity during $4 \mathrm{~h}$. All 3 conjugates have a similar binding surface and compete for the same binding site on HER2. Thus, the difference in radioactivity biodistribution and tumor binding after injection of ${ }^{111} \mathrm{In}$ -
PEP05541, ${ }^{111}$ In-PEP05838, and ${ }^{111}$ In-PEP07127 was determined by their binding sites.

The results obtained in SKOV-3-xenografted BALB/c $n u / n u$ mice are expected to reflect HER2-specific interaction of Affibody molecules with tumors and non-HER2specific interaction with normal tissues. Several previous studies indicated either that this tracer has no cross-reactivity with murine ErbB2 or that the expression of ErbB2 is negligible in mice: no decrease of the low radioactivity uptake in healthy organs of different radiolabeled derivatives of the HER2 binding $Z_{\mathrm{HER} 2: 342}$ Affibody molecule in tissues has been seen after preinjection of large amounts of nonlabeled tracer, whereas tumor uptake was saturable $(7,18)$. The slow internalization rate of HER2-binding Affibody molecules limits the effect of the prolonged retention of intracellular catabolites of the residualizing label by tumor cells, and the binding affinity (off-rate) should play a major role in the retention of conjugates in the tumor.

The results of this study showed that very high affinity does not result in higher tumor uptake in SKOV-3 xenografts with high HER 2 expression at the earlier time point ( $4 \mathrm{~h}$ after injection): no difference was seen between tracers having

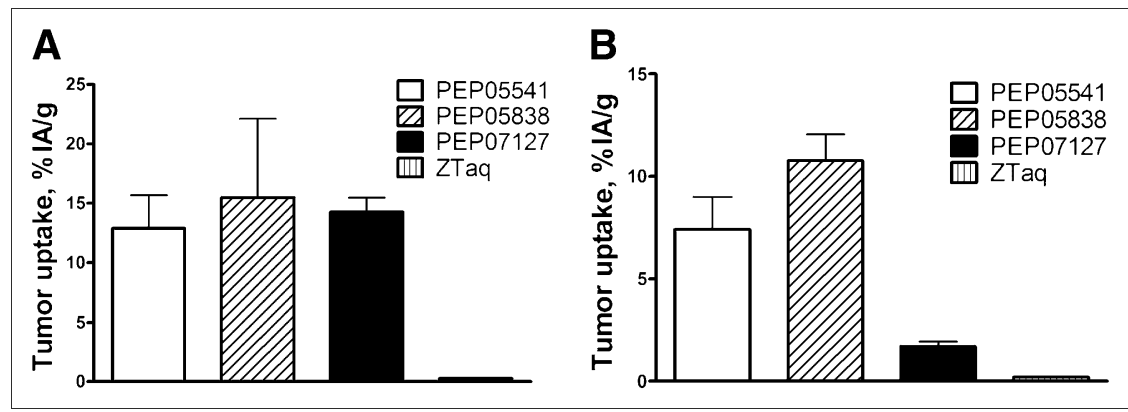

FIGURE 2. Specificity of ${ }^{111}$ In-PEP05541, ${ }^{111}$ In-PEP05838, and ${ }^{111}$ In-PEP07127 uptake in SKOV-3 (A) and LS174T (B) xenografts at $4 \mathrm{~h}$ after injection. Control group was injected with non-HER2-specific ${ }^{111}$ In$Z_{\text {Taq }}$ Affibody molecule. All animals were injected with $1 \mu \mathrm{g}$ of labeled Affibody molecules. Data are presented as average \pm SD. $\% \mathrm{IA} / \mathrm{g}=$ percentage injected radioactivity per gram of tissue. 
FIGURE 3. Tumor-to-organ ratio in BALB/C nu/nu mice bearing SKOV-3 xenografts (high level of HER2 expression) at $4 \mathrm{~h}(\mathrm{~A})$ and $24 \mathrm{~h}$ (B) after injection. Data are presented as average $(n=8)$ and SD. a = significant difference $(P<0.05)$ between ${ }^{111}$ In-PEP05541 and ${ }^{111} \mathrm{In}$-PEP05838; $\mathrm{b}=$ significant difference $(P<0.05)$ between ${ }^{111}$ In-PEP05541 and ${ }^{111}$ In-PEP07127; $\mathrm{c}=$ significant difference $(P<0.05)$ between ${ }^{111}$ In-PEP05838 and ${ }^{111} \mathrm{In}$-PEP07127.

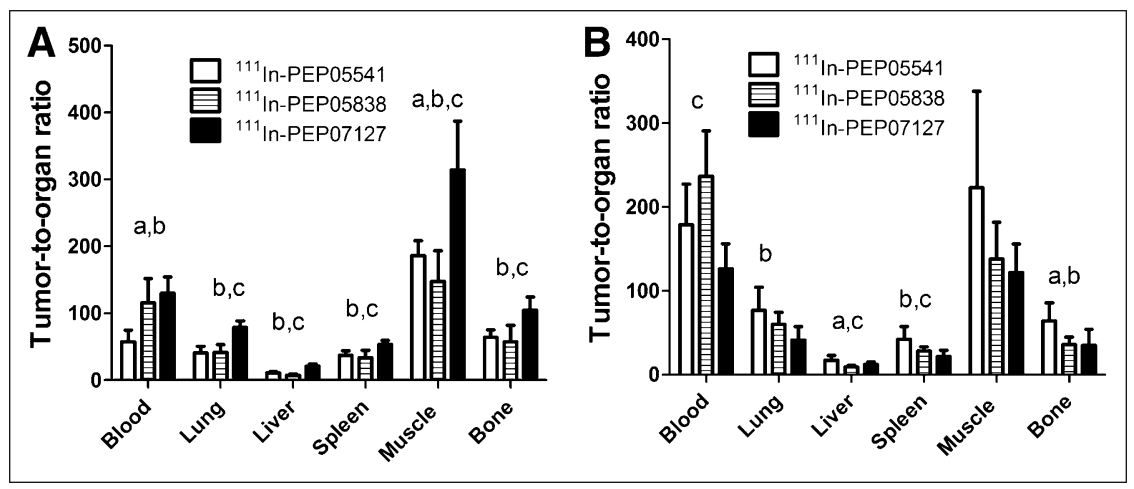

a $\mathrm{K}_{\mathrm{D}}$ of $10^{-9} \mathrm{M}$ and those having a $\mathrm{K}_{\mathrm{D}}$ of $10^{-10} \mathrm{M}$. After $24 \mathrm{~h}$, the higher dissociation rate of the moderate-affinity binder ${ }^{111}$ In-PEP07127 resulted in a lower retention of radioactivity in tumors, despite a high expression level of HER2 in the SKOV-3 tumors. These data agree well with data for HER2 binding of radioiodinated single-chain variable fragments (20), showing no difference in uptake in SKOV-3 xenografts for the tracers with affinity in the range of $10^{-9}$ to $10^{-10} \mathrm{M}$. The major difference in our experimental setting was the radionuclide, but using the nonresidualizing iodine would most likely have yielded similar results in the present study because of the slow internalization and degradation of HER2 binding Affibody molecules. The data from the present study are also in accordance with modeling studies predicting that the tumor binding of a tracer may be divided into 2 kinetic phases: a diffusionlimited loading phase and a retention phase (28). In the absence of rapid internalization, the loading phase is determined by the relation between the relative rates of intratumor diffusion and clearance, and the affinity is not important when the $\mathrm{K}_{\mathrm{D}}$ is below $10 \mathrm{nM}$. Obviously, affinity, mainly in terms of off-rate, is important in the retention phase, which is when imaging has to be performed, that is, when nonbound tracer has been cleared from normal tissues.

The picture is different in a tumor model with a much lower HER2 expression level. In this context, the accumulation of radioactivity in the tumor at $4 \mathrm{~h}$ after injection was lower for the moderate-affinity binder ${ }^{111}$ In-PEP07127 than for the 2 high-affinity binders ${ }^{111}$ In-PEP05838 and ${ }^{111} \mathrm{In}$ PEP0554. At $24 \mathrm{~h}$ after injection, the effect of lower affinity was even more pronounced. Thus, the explanation for the different results in the 2 murine models must be sought in the concentration of the HER2 target protein in the tumor tissue. The higher concentration of target protein should have different effects during the loading and retention phase, increasing the chance of binding at a given tracer concentration and increasing the chance of rebinding, respectively. Furthermore, $4 \mathrm{~h}$ after injection is past the loading phase, as the concentration of tracer in the tumor is vastly exceeding the concentration in blood. It is therefore probable that the moderate-affinity binder would yield equal tumor values in the low- and high-expressing xenografts at an earlier time point. An earlier in vitro study has shown that Affibody molecules with lower affinity penetrate more deeply into BT474 spheroids (also having a high HER2 expression) than do counterparts with a high-affinity conjugate (29). Thus, it is likely that the high-affinity binders ${ }^{111}$ In-PEP05838 and ${ }^{111}$ In-PEP05541 do not penetrate as far into the tumor. One might speculate that more target protein is available for rebinding the more deeply the tracer has traveled and that this effect may compensate for lower affinity in a context of a very high concentration of target protein, such as in a high-HER2-expressing xenograft tumor.

The results of this study demonstrate that small differences in the composition of a binding site can influence the biodistribution of HER2-binding Affibody molecules, including uptake in normal tissues. This influence may directly reflect
FIGURE 4. Tumor-to-organ ratios in BALB/ C nu/nu mice bearing LS174T xenografts (low level of HER2 expression) at $4 \mathrm{~h}(\mathrm{~A})$ and $24 \mathrm{~h}(\mathrm{~B})$ after injection. Data are presented as average $(n=6)$ and SD. $\mathrm{a}=$ significant difference $(P<0.05)$ between ${ }^{111}$ In-PEP05541 and ${ }^{111}$ In-PEP05838; $b=$ significant difference $(P<0.05)$ between ${ }^{111}$ In-PEP05541 and ${ }^{111}$ In-PEP07127; $\mathrm{c}=$ significant difference $(P<0.05)$ between ${ }^{111}$ In-PEP05838 and ${ }^{111}$ In-PEP07127.

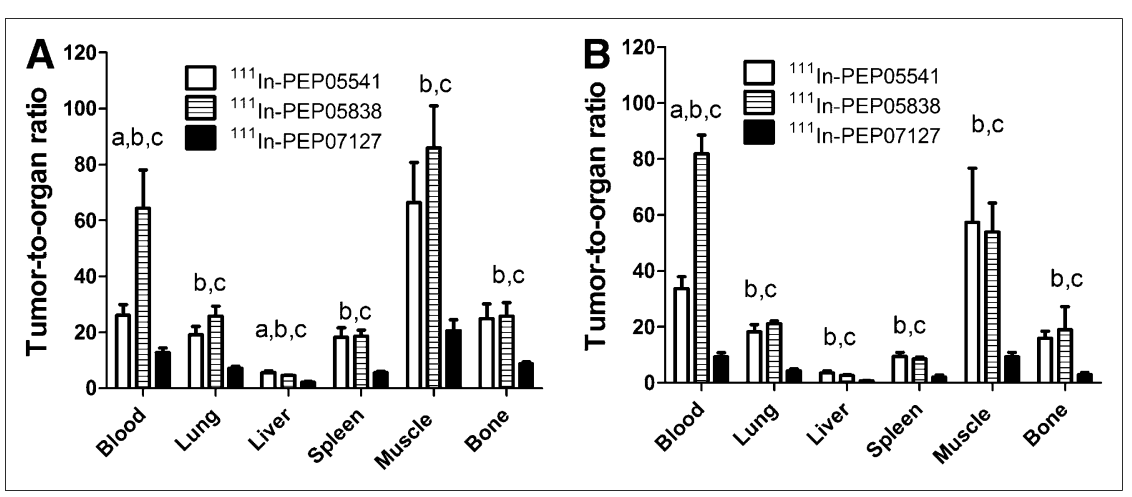


different physiochemical properties of the tracers. The few amino acids that differ between the 3 Affibody molecules are situated in the HER2-binding surface of the molecules, previously determined by x-ray crystallography (30). The Affibody molecule used in that study has a binding surface identical to the surface found in PEP05541, except for 2 peripheral amino acids not involved in binding (in positions 23 and 33). The structure showed that there is a hydrophobic spot extending the whole length of the binding site of the HER2 binder, containing M9, Y13, W14, A17, L18, I31, and Y35. In PEP07127, this hydrophobic surface is smaller because of the replacement of L18S. This difference is likely to contribute to the lower affinity of this molecule and, together with R24, makes this binder stands out as more hydrophilic than the 2 high-affinity binders. Analysis of the relative accumulation of radioactivity in various normal organs yields the same picture in both xenograft models (Tables 2 and 3). The moderate-affinity binder ${ }^{111} \mathrm{In}$ PEP07127, having the least hydrophobic binding surface, yields the lowest accumulation of radioactivity in lung, liver, spleen, bone, and muscle, whereas there is no statistical difference in renal accumulation.

The difference between the surface properties of the 2 high-affinity binders is much more subtle. Having residues $\mathrm{F}$ and $\mathrm{T}$ instead of $\mathrm{M}$ and $\mathrm{N}$ in positions 9 and 11, respectively, does make the binding surface of PEP05838 slightly more hydrophobic, whereas the replacement $\mathrm{V}$ for $\mathrm{L}$ in position 18 may be regarded as neutral. This assumption is supported by an assessment of the overall hydrophobicity of the originally randomized 13 residues of these 2 binders; applying 2 commonly used amino acids' lipophilicity scales, developed by Kyte and Doolittle (31) and Hopp and Woods (32) resulted in more lipophilic values for PEP05838 than for PEP05541. The slightly more hydrophobic binding surface is the best explanation for an increased accumulation of ${ }^{111}$ In-PEP05838 in spleen and liver, compared with the other high-affinity binder, and is in line with the more hydrophilic moderate-affinity binder ${ }^{111}$ In-PEP07127 yielding the lowest values. The presence of "lipophilic patches" on the protein surface has been reported to cause hepatic uptake (33). The intriguing feature of the present study is that all 3 HER2binders do have a hydrophobic patch in the binding surface. The higher hepatic uptake of ${ }^{111}$ In-PEP05838 is also reflected in a more rapid clearance from blood and therefore higher tumor-to-blood ratios. But from a practical point of view, accumulation of radioactivity in the liver is less desired because detection of liver metastases may be complicated. Some degree of hepatic uptake might be unavoidable with this group of high-affinity HER2-binding Affibody molecules because high affinity is generally associated with a hydrophobic binding surface (34). Not only is hydrophobicity important but, for any given group of binding proteins, the hepatic uptake may also be affected by other properties such as the isoelectric point as previously shown for Fab fragments by Kobayashi et al. (35). Clearly a much larger and more diverse data set than used in either the current study or the study by Kobayashi et al. would be needed to analyze the relative influence of hydrophobicity and isoelectric point on hepatic uptake.

\section{CONCLUSION}

Taken together, these data suggest that there is an optimal balance between hydrophobicity and affinity to be determined for each group of binders, in relation to a relevant tumor target density. Based on the properties of the 3 HER2-binding Affibody molecules in 2 xenograft models, we can conclude that an affinity in the low-nanomolar range is sufficient for efficient accumulation of a tracer in tumors with a high expression level (over $10^{6}$ receptors per cell). In the case of moderate HER2 expression, subnanomolar affinities are desirable to achieve a maximum tumor accumulation of radioactivity. These finding may be useful also in the development of Affibody molecules to other molecular targets, as well as molecular imaging agents based on other small scaffoldbased proteins.

\section{DISCLOSURE STATEMENT}

The costs of publication of this article were defrayed in part by the payment of page charges. Therefore, and solely to indicate this fact, this article is hereby marked "advertisement" in accordance with 18 USC section 1734.

\section{ACKNOWLEDGMENTS}

This work was supported by grants from the Swedish Research Council (Vetenskapsrådet) and the Swedish Cancer Society (Cancerfonden). The authors of this article, except Thuy A. Tran, previously were employed by Affibody AB, Solna, Sweden. Affibody AB holds intellectual property rights and trademarks for Affibody molecules. No other potential conflict of interest relevant to this article was reported.

\section{REFERENCES}

1. Tolmachev V, Stone-Elander S, Orlova A. Radiolabelled receptor-tyrosine-kinase targeting drugs for patient stratification and monitoring of therapy response: prospects and pitfalls. Lancet Oncol. 2010;11:992-1000.

2. Lappano R, Maggiolini M. G protein-coupled receptors: novel targets for drug discovery in cancer. Nat Rev Drug Discov. 2011;10:47-60.

3. Wester HJ, Kessler H. Molecular targeting with peptides or peptide-polymer conjugates: just a question of size? J Nucl Med. 2005;46:1940-1945.

4. Skerra A. Alternative non-antibody scaffolds for molecular recognition. Curr Opin Biotechnol. 2007;18:295-304.

5. Miao Z, Levi J, Cheng Z. Protein scaffold-based molecular probes for cancer molecular imaging. Amino Acids. 2011;41:1037-1047.

6. Löfblom J, Feldwisch J, Tolmachev V, Carlsson J, Ståhl S, Frejd FY. Affibody molecules: engineered proteins for therapeutic, diagnostic and biotechnological applications. FEBS Lett. 2010;584:2670-2680.

7. Orlova A, Magnusson M, Eriksson TL, et al. Tumor imaging using a picomolar affinity HER2 binding affibody molecule. Cancer Res. 2006;66:4339-4348.

8. Friedman M, Orlova A, Johansson E, et al. Directed evolution to low nanomolar affinity of a tumor-targeting epidermal growth factor receptor-binding affibody molecule. J Mol Biol. 2008;376:1388-1402.

9. Li J, Lundberg E, Vernet E, Larsson B, Höidén-Guthenberg I, Gräslund T. Selection of affibody molecules to the ligand-binding site of the insulin-like growth factor-1 receptor. Biotechnol Appl Biochem. 2010;55:99-109.

10. Lindborg M, Cortez E, Höidén-Guthenberg I, et al. Engineered high-affinity affibody molecules targeting platelet-derived growth factor receptor $\beta$ in vivo. J Mol Biol. 2011;407:298-315. 
11. Ahlgren S, Tolmachev V. Radionuclide molecular imaging using Affibody molecules. Curr Pharm Biotechnol. 2010;11:581-589.

12. Baum RP, Prasad V, Müller D, et al. Molecular imaging of HER2-expressing malignant tumors in breast cancer patients using synthetic ${ }^{111} \mathrm{In}$ - or ${ }^{68} \mathrm{Ga}$-labeled affibody molecules. J Nucl Med. 2010;51:892-897.

13. Rudnick SI, Adams GP. Affinity and avidity in antibody-based tumor targeting. Cancer Biother Radiopharm. 2009;24:155-161.

14. Schmidt MM, Wittrup KD. A modeling analysis of the effects of molecular size and binding affinity on tumor targeting. Mol Cancer Ther. 2009;8:2861-2871.

15. Tolmachev V, Orlova A. Influence of labelling methods on biodistribution and imaging properties of radiolabelled peptides for visualisation of molecular therapeutic targets. Curr Med Chem. 2010;17:2636-2655.

16. Wållberg H, Orlova A. Slow internalization of anti-HER2 synthetic affibody monomer ${ }^{111}$ In-DOTA-ZHER2:342-pep2: implications for development of labeled tracers. Cancer Biother Radiopharm. 2008;23:435-442.

17. Ahlgren S, Orlova A, Rosik D, et al. Evaluation of maleimide derivative of DOTA for site-specific labeling of recombinant affibody molecules. Bioconjug Chem. 2008; 19:235-243.

18. Tran TA, Rosik D, Abrahmsén L, et al. Design, synthesis and biological evaluation of a multifunctional HER2-specific Affibody molecule for molecular imaging. Eur J Nucl Med Mol Imaging. 2009;36:1864-1873.

19. Adams GP, Schier R, Marshall K, et al. Increased affinity leads to improved selective tumor delivery of single-chain Fv antibodies. Cancer Res. 1998;58:485-490.

20. Adams GP, Schier R, McCall AM, et al. High affinity restricts the localization and tumor penetration of single-chain $\mathrm{fv}$ antibody molecules. Cancer Res. 2001;61:4750-4755.

21. Adams GP, Tai MS, McCartney JE, et al. Avidity-mediated enhancement of in vivo tumor targeting by single-chain Fv dimers. Clin Cancer Res. 2006;12:1599-1605.

22. Fujimori K, Covell DG, Fletcher JE, Weinstein JN. Modeling analysis of the global and microscopic distribution of immunoglobulin $\mathrm{G}, \mathrm{F}\left(\mathrm{ab}^{\prime}\right) 2$, and Fab in tumors. Cancer Res. 1989;49:5656-5663.

23. Minner S, Jessen B, Stiedenroth L, et al. Low level HER2 overexpression is associated with rapid tumor cell proliferation and poor prognosis in prostate cancer. Clin Cancer Res. 2010;16:1553-1560.
24. Tolmachev V, Hofström C, Malmberg J, et al. HEHEHE-tagged affibody molecule may be purified by IMAC, is conveniently labeled with $\left[{ }^{99 \mathrm{~m}} \mathrm{Tc}(\mathrm{CO})_{3}\right](+)$, and shows improved biodistribution with reduced hepatic radioactivity accumulation. Bioconjug Chem. 2010;21:2013-2022.

25. Hofström C, Orlova A, Altai M, Wångsell F, Gräslund T, Tolmachev V. Use of a HEHEHE purification tag instead of a hexahistidine tag improves biodistribution of Affibody molecules site-specifically labeled with ${ }^{99 \mathrm{~m}} \mathrm{Tc},{ }^{111} \mathrm{In}$, and ${ }^{125} \mathrm{I}$. J Med Chem. 2011;54:3817-3826.

26. Malmberg J, Tolmachev V, Orlova A. Imaging agents for in vivo molecular profiling of disseminated prostate cancer: cellular processing of $\left[{ }^{111} \mathrm{In}\right]$-labeled CHX-A" DTPA-trastuzumab and anti-HER2 ABY-025 Affibody in prostate cancer cell lines. Experiment Ther Med. 2011;2:523-528.

27. De León-Rodríguez LM, Kovacs Z. The synthesis and chelation chemistry of DOTA-peptide conjugates. Bioconjug Chem. 2008;19:391-402.

28. Graff CP, Wittrup KD. Theoretical analysis of antibody targeting of tumor spheroids: importance of dosage for penetration, and affinity for retention. Cancer Res. 2003;63:1288-1296.

29. Qvarnström OF, Simonsson M, Carlsson J, Tran TA. Effects of affinity on binding of HER2-targeting Affibody molecules: model experiments in breast cancer spheroids. Int J Oncol. 2011;39:353-359.

30. Eigenbrot C, Ultsch M, Dubnovitsky A, Abrahmsén L, Härd T. Structural basis for high-affinity HER2 receptor binding by an engineered protein. Proc Natl Acad Sci USA. 2010;107:15039-15044.

31. Kyte J, Doolittle RF. A simple method for displaying the hydropathic character of a protein. J Mol Biol. 1982;157:105-132.

32. Hopp TP, Woods KR. Prediction of protein antigenic determinants from amino acid sequence. Proc Natl Acad Sci USA. 1981;78:3824-3828.

33. Rusckowski M, Qu T, Gupta S, Ley A, Hnatowich DJ. A comparison in monkeys of ${ }^{99 \mathrm{~m}}$ Tc labeled to a peptide by 4 methods. J Nucl Med. 2001;42:1870-1877.

34. Kuntz ID, Chen K, Sharp KA, Kollman PA. The maximal affinity of ligands. Proc Natl Acad Sci USA. 1999;96:9997-10002.

35. Kobayashi H, Le N, Kim IS, et al. The pharmacokinetic characteristics of glycolated humanized anti-Tac Fabs are determined by their isoelectric points. Cancer Res. 1999;59:422-430. 